\title{
Detection of $m c r-4$ positive Salmonella enterica serovar Typhimurium in clinical isolates of human origin, Italy, October to November 2016
}

Edoardo Carretto ${ }^{1}$, Flavia Brovarone ${ }^{1}$, Paola Nardini ${ }^{1}$, Giuseppe Russello ${ }^{1}$, Daniela Barbarini², Stefano Pongolini ${ }^{3}$, Carlo

Gagliotti ${ }^{4}$, Alessandra Carattoli5, Mario Sarti ${ }^{6}$

1. Clinical Microbiology Laboratory, IRCCS Arcispedale Santa Maria Nuova, Reggio Emilia, Italy

2. Clinical Virology and Microbiology Laboratory - Fondazione IRCCS Policlinico San Matteo, Pavia, Italy

3. Risk Analysis Unit, Istituto Zooprofilattico Sperimentale della Lombardia e dell’Emilia-Romagna, Sezione di Parma, Parma, Italy

4. Regional Health and Social Agency of Emilia-Romagna, Bologna, Italy

5. Department of Infectious Diseases, Istituto Superiore di Sanità, Rome, Italy

6. Clinical Microbiology Laboratory, S. Agostino-Estense Hospital, Baggiovara, Italy

Correspondence: Edoardo Carretto (edoardo.carretto@ausl.re.it)

Citation style for this article:

Carretto Edoardo, Brovarone Flavia, Nardini Paola, Russello Giuseppe, Barbarini Daniela, Pongolini Stefano, Gagliotti Carlo, Carattoli Alessandra, Sarti Mario. Detection of mcr-4 positive Salmonella enterica serovar Typhimurium in clinical isolates of human origin, Italy, October to November 2016 . Euro Surveill.

2018;23(2): pii=17-00821. https://doi.org/10.2807/1560-7917.ES.2018.23.2.17-00821

Article submitted on 06 Dec 2017 / accepted on 11 Jan 2018 / published on 11 Jan 2018

In this study we report the detection of the recently described $m c r-4$ gene in two human isolates of Salmonella enterica serovar Typhimurium. The strains were isolated from faecal samples of two Italian patients with gastroenteritis, collected in 2016. The identified $m c r-4$ genes (variant $m c r-4.2)$ differed from the $m c r-4$ gene originally described in a Salmonella strain of swine origin from Italy. Salmonella species could represent a hidden reservoir for mor genes.

In the context of an analysis into the epidemiology of mobile colistin determinants, we investigated a collection of 106 human clinical isolates of Enterobacteriaceae. These isolates were obtained between January 2016 and October 2017 in two main hospitals in Emilia Romagna, Italy, namely IRCCS Arcispedale Santa Maria Nuova, Reggio Emilia and Sant'Agostino-Estense Hospital of Baggiovara. These isolates had originally been selected on the basis of their reduced susceptibility to colistin (MIC $\geq 2 \mathrm{mg} / \mathrm{L}$ ). Among the 67 Escherichia coli, 27 Klebsiella pneumoniae, six Salmonella species and six other Enterobacteriaceae collected, we detected the mcr-4 gene in two human isolates of $S$. enterica subsp. enterica monophasic variant of serovar Typhimurium. The $\mathrm{mcr}-4$ gene was not detected in any other isolate of the above collection; further analyses for other mor genes are ongoing in these and additional isolates.

\section{Description of the isolates}

The two Salmonella strains were isolated from faecal samples of outpatients with gastroenteritis who were referred to the Sant'Agostino-Estense Hospital. The first specimen, named $A B-160$, was collected in
October 2016, the second one (AB-243) in November 2016. Patient data did not reveal any social, nosocomial or epidemiological links between the two patients. The disease was self-limiting and did not require hospitalisation or supportive therapy. According to European Committee on Antimicrobial Susceptibility Testing (EUCAST) breakpoints [1], the two isolates showed the same susceptibility profile on an automated instrument (Vitek-2, BioMérieux, France) and were resistant to ampicillin, piperacillin and colistin and susceptible to other beta-lactams.

After their isolation, the strains were serotyped by the Istituto Zooprofilattico Sperimentale della Lombardia e dell'Emilia-Romagna (IZSLER) following the WhiteKauffmann-Le Minor scheme by slide agglutination with $\mathrm{O}$ and $\mathrm{H}$ antigen-specific sera (DID, Milan, Italy; Biogenetics, Padua, Italy). Both strains were identified as S. enterica subsp. enterica monophasic variant of serovar Typhimurium with antigenic formula 4,[5],12:i:[2]. PFGE was performed according to the PulseNet protocol with $\mathrm{Xbal}$ digestion [3], and restriction profiles were analysed by BioNumerics 7.5 (Applied Maths, Saint-Martens - Latem, Belgium). The two isolates had different pulsotypes (AB-160: STYMXB.0083 and AB-243: STYMXB_PR.1681) but those were very similar, with a single band difference.

Colistin resistance was initially detected by using the automated instrument Vitek-2 (BioMérieux, France). They were confirmed in two different centres by using BMD-based commercial systems (SensiTest Colistin, Liofilchem, Italy, and Sensititre, ThermoFisher, US) and also with BMD performed in the IRCCS Arcispedale Santa Maria Nuova of Reggio Emilia according to the 
Clinical and Laboratory Standards Institute (CLSI) [4] and EUCAST (ISO standard method 20776-1) [5]. Three reference strains ( $E$. coli ATCC 25922, Pseudomonas aeruginosa ATCC 27853 and E. coli NCTC 13846) were used as controls for susceptibility testing. Both Salmonella strains had a MIC for colistin of $8 \mathrm{mg} / \mathrm{L}$ by using SensiTest Colistin and the reference BMD, whereas Sensititre documented MICs of $4 \mathrm{mg} / \mathrm{L}$ and 8 $\mathrm{mg} / \mathrm{L}$ for isolate $A B-160$ and $A B-243$, respectively.

\section{Detection of $m c r-4$}

The DNA of the isolates was extracted using UltraClean microbial DNA isolation kit (MoBio Laboratories, US). The presence of $\mathrm{mcr}$ genes was investigated by different PCRs with previously described primers [6-10]. Both Salmonellaisolates were positive for the $\mathrm{mcr}-4$ gene, using the internal primers Mcr-4 FW and Mcr-4 RV [7], and negative for the other four $\mathrm{mcr}$ genes. On these two isolates, the external primers Mcr-4 ext FW and Mcr-4 ext RV [7], targeting a fragment between 104 bases upstream of the start codon and 91 bases downstream of the stop codon of the mcr-4gene, gave no positive amplification. Furthermore, primers targeting the repB gene of the ColE1o plasmid did not give any amplification product [7].

To better characterise these $m c r-4$ genes, we designed two additional primers derived from the sequence of the $m c r$-4gene published in GenBank, accession number NG_055659.1. The forward primer was Mcr4Fw_Out (AATGAGGTCAAGCTAGTAT) corresponding to bases 54-72 of NG_055659.1 (located at 47 to 29 bases upstream of the $\mathrm{mcr}-4$ start codon), and the reverse primer was Mcr4Rv_Out (ATATGTCACCCCTAAGATAA), matching bases $1,744-1,763$ of NG_055659.1 (located at 18 to 37 bases downstream of the $\mathrm{mcr}-4$ stop codon). The annealing temperature was $54^{\circ} \mathrm{C}$.

An amplification product was obtained for both isolates and sequenced using both the above primers and previous internal primers. A sequence of 1,630 bp, identical for the two isolates, was obtained and deposited in GenBank with the accession number MG581979. MG581979 was a perfect match with the sequence NG_055659.1 across 1,629 bp, with a single nucleotide polymorphism (SNP) at position 1,001 of MG581979 (A to $G)$, causing a transition of $Q$ to $R$ at amino acid position 331. This mutation defines this allele which we refer to as $m c r-4.2$.

\section{Discussion}

Colistin is currently considered as the last-choice treatment for human infection caused by multidrugresistant Gram-negative bacteria, despite well documented side effects and toxicity. Until recently, colistin resistance was extremely rare, mostly because of chromosomal mutations. In 2016, the first plasmidmediated resistance to colistin was reported with the discovery of the mcr-1 gene which encodes a phosphoethanolamine transferase [10]. Since then, different studies, based on retrospective analysis of strain collections, have demonstrated that this gene has a worldwide distribution [11], suggestive of a period of undetected dissemination. Currently, at least 12 different $m c r$-1gene variants have been acknowledged in GenBank (from mcr-1.1 to mcr-1.12: KP347127.1; KX236309.1; KY400027.1; KY041856.1; KY271416.1; KY352406.1; KY488488.1; KY685070.1; KY685071.1; MF176238.1; KY853650.1; LC337668.1). Furthermore, four additional $\mathrm{mcr}$ genes with varying homology to $\mathrm{mcr}_{-1}$ have been discovered and named $\mathrm{mcr}-2$, $\mathrm{mcr}$ 3, mcr-4 and mcr-5 [6-9]. The mcr-4 gene was first described in 2017 in an Italian strain of Salmonella enterica subsp. enterica (monophasic variant of serovar Typhimurium 4,[5],12, i:-) of swine origin isolated in 2013 [7].

To our knowledge, this is the first report of $\mathrm{mcr}$ 4-positive bacterial isolates of human origin. The two Salmonellaspecies belonged to the same serovar as the first mcr-4-positive Salmonella strain reported in a pig slaughtered in Italy [7]. That study had also reported 11 mcr-4-positive $E$. coli strains of animal origin in Spain and in Belgium, suggesting a dissemination of the novel gene among European countries and highlighting the need to evaluate possible risk and burden for human health [7]. The presence of the different $m c r-4.2$ variant, the lack of amplification with external primers located outside of the coding sequence and the negative result for the ColE10 plasmid suggest that the genetic background of the $\mathrm{mcr}-4$ gene in our two human isolates is different from that in the Salmonella R3445 of animal origin [7]. Further analysis through whole genome sequencing is in progress to investigate the genetic homology of the two human isolates and their phylogenetic relatedness with the Salmonella isolate of veterinary origin.

This serovar of Salmonella is involved in most cases of human infection in the Emilia-Romagna region (personal communication: Stefano Pongolini, regional reference laboratory for surveillance of enteric pathogens at IZSLER, July 2017). Particularly in the period from July to August 2014, pulsotype STYMXB.0083 spread extensively thorough the provinces of Reggio Emilia and Modena (personal communication: Stefano Pongolini, IZSLER section of Parma, July 2017). On that occasion, the source of infection was identified as a pork product contaminated with the same Salmonella genotype as the human isolates. Since one of our strains had the pulsotype STYMXB.0083, further studies on the possible spread of mobile colistin resistance in a larger set of Salmonella strains, at least in our area, would be helpful to assess the risk to human health.

As demonstrated by the wide distribution of mcr-1 worldwide [11], it could be hypothesised that also $m c r-4$ has been spreading for many years undetected among Enterobacteriaceae in animals in European countries, with occasional transmission to humans. Rates of colistin resistance could be underestimated owing to the poor performance of different 
automated systems used routinely in clinical microbiology laboratories $[12,13]$. Epidemiology and distribution of $\mathrm{mor}$ genes among animal and human isolates should be further investigated to evaluate their dissemination and possible transmission to strains which harbour other resistance determinants, such as extendedspectrum beta-lactamases and carbapenemases, as already demonstrated [12]. Acquisition of mobile colistin resistance by multidrug-resistant microorganisms in clinical settings would represent a serious problem for treatment and infection control [14]. Salmonella could represent a hidden reservoir for mobile colistin resistance determinants, considering the intensive use of colistin in animal farming that may have contributed to the selection of colistin-resistant strains [7]. Further studies and implementation of surveillance policies (improvement of laboratory methods for correct evaluation of phenotypical colistin resistance, e.g. by using BMD-based techniques as first-line antimicrobial susceptibility testing, and epidemiological analyses at local and national level, based on molecular detection of the different mobile resistance determinants, e.g. by multiplex PCRs) may be useful to understand the complexity and extent of the circulation of the different $\mathrm{mcr}$ genes and their impact in human health [15].

\section{Acknowledgements}

We are grateful to Dr Emilio Scotti for his helpful technical support in sequencing procedures. The authors thank $\mathrm{Dr}$ Jonathan $\mathrm{M}$ Tyrrell for his support in revising the manuscript.

\section{Conflict of interest}

None declared.

\section{Authors' contributions}

This study was designed by EC and AC. Isolates were collected by MS. Experimental work was done by EC, FB, GR, DB, SP. Data were analysed and interpreted by EC, DB, SP and $A C$. The manuscript was drafted by EC and PN, revised by EC, $A C$ and SP and was reviewed by all authors.

References

1. The European Committee on Antimicrobial Susceptibility Testing (EUCAST). Breakpoint tables for interpretation of MICS and zone diameters. Version 7.1, 2017. Växjö: EUCAST; 2017. Available from: http://www.eucast.org/fileadmin/src/media/ PDFs/EUCAST_files/Breakpoint_tables/v_7.1_Breakpoint_ Tables.pdf

2. International Organization for Standardization (ISO). Microbiology of the food chain. Horizontal method for the detection, enumeration and serotyping of Salmonella. Part 3: Guidelines for serotyping of Salmonella spp. ISO/TR 6579 3:2014. Geneva: ISO; 2014. Available from: https://www.iso. org/standard/56714.html

3. Centre for Disease Control and Prevention (CDC). Standard operating procedure for PulseNet PFGE of Escherichia coli 0157: H7, Escherichia coli non-0157 (STEC), Salmonella serotypes, Shigella sonnei and Shigella flexneri. Atlanta: CDC; 2013. Available from: http://www.cdc.gov/pulsenet/pdf/ecolishigella-salmonella-pfge-protocol-508c.pdf

4. Clinical Laboratory Standard Institute (CLSI). Performance Standards for Antimicrobial Susceptibility Testing. 27th ed. CLSI supplement M100S. Wayne, PA: CLSI; 2017. Available from: https://clsi.org/standards/products/microbiology/ documents/m100/

5. International Organization for Standardization (ISO). ISO20776-1:2006 Clinical laboratory testing and in vitro diagnostic test systems - Susceptibility testing of infectious agents and evaluation of performance of antimicrobial susceptibility test devices - Part 1: Reference method for testing the in vitro activity of antimicrobial agents against rapidly growing aerobic bacteria involved in infectious diseases. Geneva: ISO; 2006. Available from: https://www.iso.org/standard/41630.html

6. Borowiak M, Fischer J, Hammerl JA, Hendriksen RS, Szabo I, Malorny B. Identification of a novel transposon-associated phosphoethanolamine transferase gene, mcr-5, conferring colistin resistance in d-tartrate fermenting Salmonella enterica subsp. enterica serovar Paratyphi B. J Antimicrob Chemother. 2017;72(12):3317-24. https://doi.org/10.1093/jac/dkx327 PMID: 28962028

7. Carattoli A, Villa L, Feudi C, Curcio L, Orsini S, Luppi A, et al. Novel plasmid-mediated colistin resistance mcr-4 gene in Salmonella and Escherichia coli, Italy 2013, Spain and Belgium, 2015 to 2016. Euro Surveill. 2017;22(31):30589. https://doi.org/10.2807/1560-7917.ES.2017.22.31.30589 PMID: 28797329

8. Xavier BB, Lammens C, Ruhal R, Kumar-Singh S, Butaye P, Goossens $\mathrm{H}$, et al. Identification of a novel plasmid-mediated colistin-resistance gene, mcr-2, in Escherichia coli, Belgium, June 2016. Euro Surveill. 2016;21(27):30280. https://doi. org/10.2807/1560-7917.ES.2016.21.27.30280 PMID: 27416987

9. Yin W, Li H, Shen Y, Liu Z, Wang S, Shen Z, et al. Novel plasmid-mediated colistin resistance gene mcr-3 in Escherichia coli. MBio. 2017;8(3):e00543-17. https://doi.org/10.1128/ mBio.00543-17 PMID: 28655818

10. Liu YY, Wang Y, Walsh TR, Yi LX, Zhang R, Spencer J, et al. Emergence of plasmid-mediated colistin resistance mechanism MCR-1 in animals and human beings in China: a microbiological and molecular biological study. Lancet Infect Dis. 2016;16(2):161-8. https://doi.org/10.1016/S14733099(15)00424-7 PMID: 26603172

11. Schwarz S, Johnson AP. Transferable resistance to colistin: a new but old threat. J Antimicrob Chemother. 2016;71(8):2066 70. https://doi.org/10.1093/jac/dkw274 PMID: 27342545

12. Chew KL, La MV, Lin RTP, Teo JWP. Colistin and polymyxin B susceptibility testing for carbapenem-resistant and $\mathrm{mcr}$ positive Enterobacteriaceae: comparison of Sensititre, MicroScan, Vitek 2, and Etest with broth microdilution. J Clin Microbiol. 2017;55(9):2609-16. https://doi.org/10.1128/ JCM.00268-17 PMID: 28592552

13. European Committee on Antimicrobial Susceptibility Testing (EUCAST). Antimicrobial susceptibility testing of colistin problems detected with several commercially available products. In: EUCAST warnings concerning antimicrobial susceptibility testing products or procedures. Växjö: EUCAST; 2017. Available from: http://www.eucast.org/ast_of_bacteria/ warnings/

14. Delgado-Blas JF, Ovejero CM, Abadia-Patiño L, Gonzalez-Zorn B. Coexistence of mcr-1 and blaNDM-1 in Escherichia coli from Venezuela. Antimicrob Agents Chemother. 2016;60(10):6356-8. https://doi.org/10.1128/AAC.01319-16 PMID: 27431212

15. European Centre for Disease Prevention and Control (ECDC). Plasmid-mediated colistin resistance in Enterobacteriaceae. Stockholm: ECDC; 2016. Available from: https://ecdc.europa. eu/sites/portal/files/media/en/publications/Publications/ enterobacteriaceae-risk-assessment-diseases-caused-byantimicrobial-resistant-microorganisms-europe-june-2016.pdf

\section{License and copyright}

This is an open-access article distributed under the terms of the Creative Commons Attribution (CC BY 4.0) Licence. You may share and adapt the material, but must give appropriate credit to the source, provide a link to the licence, and indicate if changes were made.

This article is copyright of the authors, 2018. 